\title{
Artificial intelligence (AI) in breast cancer care - Leveraging multidisciplinary skills to improve care ${ }^{\text {is }}$
}

Artificial Intelligence (AI) technologies supporting health professionals' skills bring the promise of an era in which repetitive and time-intensive tasks in healthcare can be automated and performed (either entirely or in part) by AI systems. As automation progressively facilitates the delivery of health care, physicians can now focus on more humanized patient care. The use of AI to ensure quality delivery is essential in the fight against diseases and there is no doubt that the next decade will see a growing stream of AI applications across healthcare. "We are not ready for what is about to come" Coiera tells us [1], a statement highlighting the need for healthcare practitioners and services to prepare for Al's adoption into health practice. With the progress of AI systems, it seems obvious that machine thinking will invade our workspace, literally and figuratively, in all areas of breast cancer care. Instead of resisting, we should better consider preparing ourselves for the impact, the potential, and the pitfalls, and start adapting our work environment to this new reality. This progress will need big input from "both sides of the barricade", namely AI technical experts and health care professionals.

Fear of the unknown is a common obstacle in innovation and frequently leads to resistance to new approaches. This is also the situation with AI in health care, although in this instance, cautious planning and evaluation are needed to allow us to understand the most beneficial way to adopt $\mathrm{AI}$ in practice. To achieve this, healthcare professionals including doctors need to work closely with AI experts. However, many doctors are not at ease with the computer science language, and computer scientists and engineers don't master health terminology and concepts. Moreover, these very divergent skills appear to focus on different objectives. While health professionals have the primary goal of better diagnosis and treatment for patients, computer science teams are focused on inventing methods and computational processes to solve questions that might, in some instances, be considered of less priority to those who actually manage patients in real-word health settings. So, to make progress in our endeavor to use AI to improve various aspects of clinical care, in general and in our focus on breast cancer, we need to communicate our different perspectives more efficiently and more effectively. To help solve this problem, a curriculum teaching the language used and the problems addressed both by AI scientists and healthcare professionals could be implemented both in pre-graduate and post-graduate education, as highlighted in the work from Ferreira et al. in this special issue [2].

Many of the subfields of AI in health care are represented in this

\footnotetext{
This paper has been co-published in the BREAST and Artificial Intelligence in Medicine.
}

collection of papers addressing some of the developments that will help us in the routine care of breast cancer patients. It is obvious that many of the tasks that are undertaken very frequently by human operators with their inherent subjectivity will be the ones most likely to be replaced successfully in the near future by AIbased systems.

One of the most relevant fields of research is the use of image analysis in various clinical applications including breast imaging, digital pathology, surgical planning, and outcomes evaluation. The huge amount of imaging digital data already annotated with defined characteristics either in screening or diagnosis has allowed the development of machine-learning-derived solutions that are on the verge of becoming a part of our practices in the near future.

On the methodological side, contributions to this special issue come from the papers by Ref. [3,4]. Yap et al. move from a set of ultrasound breast images and use an accurate object detection deep learning framework (namely, Faster-RCNN with Inception-ResNetv2): region of interest (ROI) detection and lesion localization are automatically performed, helping the surgeon to focus on the relevant area of the breast. Carvalho et al. propose a method which deploys phylogenetic diversity indexes, generally used in biology to compare the behavior patterns of species in different areas, applied in this work to characterize breast histopathology images: the resulting model classifies histopathological images of the breast into four classes - invasive carcinoma, in situ carcinoma, normal tissue, and benign lesion - leaving a possible promise for the future of reducing the number of needed biopsies.

Other papers in this special issue are also dedicated to the use of $\mathrm{AI}$ in breast imaging, screening, diagnosis, and prognosis as well as the prediction of cancer response to treatment. These topics are reviewed and discussed taking into account the current state of existing applications [5-8]. AI has the potential to bring efficiencies to screening programs burdened by screen-reading workload, and augmenting radiologists' interpretation. Various approaches to using $\mathrm{AI}$ in screening are discussed by Sechopoulos and Mann in this issue [7], while Lee et al. highlight the pathways and processes needed to ensure validation and diversification of algorithms for screening practice [5].

Applications of machine learning to digital pathology for breast cancer, including both diagnostic and prognostic applications, will not only complement the routine work of breast pathologists but also improve their diagnostic accuracy. As described in the detailed review by Ibrahim et al. [9], AI in breast cancer pathology will provide information beyond that can be gained by eyeball assessment with the potential to replace some of the expensive multigene assays.

In contrast to imaging and pathology where there are already 
available AI tools and substantial applied research, the subspecialties in local treatments of breast cancer are relatively lagging behind in AI applications. It is not surprising that AI work in surgery is not commensurate with that described for imaging and pathology. Operating a breast depends on a number of variables that are difficult to collect and standardize. An effort has been made to try to transfer the diagnosis imaging information to the operating room, allowing more accurate localization of cancer. This effort has been essentially directed to the fusion of information coming from surface scans with imaging data from MRI allowing the construction of a breast model with the tumor inside, as reported by Bessa et al. [10]. Some work has also been done in breast reconstruction through image analysis of angio CTs, the routine exam used to plan free flap reconstruction after mastectomy. The capacity to automatically detect vessels from angio CTs, proposed by Mavioso et al. will hopefully replace the process of manual annotation by a computerized automatic analysis keeping the same accuracy levels [11].

Concerning radiotherapy, CT images are the core of treatment planning for each patient, and some facilitating tools already exist for contouring the fields of treatment. However, the use of AI to automate the entire process is not yet used in breast cancer although it could save many hours of work for the radiation oncology team. The paper from Poortmans et al. offers a significant contribution to elucidate the issues related to AI applications to individualize radiation therapy for breast cancer patients [12].

Beyond using image analysis in diagnostic and planning of treatment, we also need tools to assess the clinical outcomes of this decision. Besides the side effects of systemic treatments, the visible impact of surgery and radiotherapy on patients' body image is of major importance for patients. Cardoso and colleagues [13] offer a review and a discussion of recent advancements in machine learning, and especially deep learning, bringing new promises to the field of aesthetic outcomes of locoregional treatments.

Decision support systems are advanced systems that help physicians to diagnose and treat diseases.

In fact, the task of physicians is becoming more and more complex because increasing quantities of data need to be properly considered before proceeding with any treatment decision. Indeed, because medical knowledge is advancing, different categories of cancer are proliferating along with the number of new therapies stemming from clinical research and new surgery techniques, which dramatically widen the set of possible choices of decisions. Decision support systems make use of different methodologies including artificial intelligence, knowledge representation, information visualization, and text mining, among others. On the methodological side, contributions on decision support systems come from the papers by Ref. [14,15]. Bouaud et al. report about the results of the European-funded DESIREE project, providing breast unit physicians with different complementary therapeutic decision support modules including guideline-based, experience-based, and case-based reasoning. Gu et al. propose an auxiliary decision support system to help doctors improve the accuracy of breast cancer recurrence prediction.

Big data analysis refers to the analysis of huge quantities of data which are now collected and stored in any human activity, including healthcare. Such data can be exploited for different targets, e.g., to provide medical staff with suggestion support for care delivery, to reason, and to extract knowledge, as well as to understand the natural language of textual descriptions such as medical reports.

Papers by Macias-Garcia et al. [16], and Pozzoli et al. [17] offer significant contributions to the methodological side of the big data topic. Macias-Garcia et al. summarize DNA methylation data to generate new features from the values of $\mathrm{CpG}$ sites of patients, to predict breast cancer recurrence. Pozzoli et al. experiment unsupervised learning in the detection and analysis of distinctive proteins for the identification of cancer subtypes.

Natural language processing (NLP) refers to the capacity of analyzing free text, i.e., the text in human normal speaking or writing, and of understanding it and, possibly, of extracting knowledge from it. The main sources for NLP are medical reports, where non-coded information is depicted. On the methodological side, one contribution on NLP comes from the paper by Hanyin Wang et al. [18], where the authors propose an automated method to predict breast cancer distant recurrence, using natural language processing and deep learning approaches applied to pathology reports and progress notes of some 6000 patients.

Some less established but however emerging and promising areas of AI applications to breast cancer care are also introduced into this issue.

Butow and Hoque [19] discuss AI use to assess and define effective communication between health professionals and patients. Both communication and teaching of how to communicate are processes that stand essentially on qualitative forms of analysis/evaluation, and the use of machine learning could make these processes more efficient.

Moser and Nayaran [20] report on the use of machine learning to detect patterns that help to track the ideal workflow for breast cancer patients from diagnosis to long-term follow-up. The authors propose that electronic records analysis could bring AI modeling into clinical practice to drive well-coordinated, patient-centered cancer care in the complex web of modern healthcare today, yet stressing the need for human presence along these pathways.

Kakileti et al. [21] propose and analyze a new personalized risk framework to identify a high-risk target population for regular screening and enable early stage breast cancer detection at scale. The approach runs machine learning algorithms to analyze thermal images, and automatically generates a breast health risk score, assigning subjects to four different risk classes.

Chiudinelli et al. [22] analyze data extracted from some 3000 electronic health records and describe a care flow mining algorithm to define the most frequent patterns of care. The results allow drawing electronic temporal phenotypes which describe wellcharacterized pathology classes across the studied population, and provide the surgeons with relevant help in care delivery.

A further crucial topic for applying AI in breast cancer care, and indeed relevant for any application of AI in healthcare, relates to ethical, legal, and social issues (ELSI), wonderfully addressed in the paper by Carter and colleagues [23], in this special issue. This highlights the ELSI challenges and their possible solutions and reinforces the importance of thinking carefully on what is to come and addressing AI risks before implementation. This approach will have to include all stakeholders involved to the design an effective risk mitigation plan that will support the introduction of $\mathrm{AI}$ in breast cancer care and inform its expansion in ethical and socially acceptable pathways.

$\mathrm{AI}$ is here to stay, generally in healthcare, but also specifically in breast cancer care. Structured research is needed to investigate not only the accuracy and utility of the new AI-related processes compared to what exists as standard of care, but also to ensure these AI tools are transferable beyond the specific settings where they were developed. The future could change rapidly and the way we adapt to it will define how effectively multidisciplinarity operates and how clinical skills work jointly with AI skills to develop the pathway for implementing AI in clinical care for the benefit of breast cancer patients. Such a pathway must consider an ethical construct for using AI and engage stakeholders, including breast cancer consumers, in decisions regarding where AI is acceptable as part of healthcare. This may enable us to build a future 
where AI will be used to augment not only healthcare professionals but also patients, to inform or complement, rather than dictate clinical decisions, and to ultimately transition to an AI-supported clinical pathway in breast cancer care.

\section{Online-only supplementary Appendix: (Link xxxx)}

Methodological issues: The idea of having a virtual joint special issue on the advent of Artificial Intelligence (AI) in the management of breast cancer was launched by the publisher at the end of 2018 . The special issue would have to be joint, i.e. collecting contributions on the same topic coming from two different journals. Indeed, each journal, with its specific target scientists, research field, and research methods, would have addressed only some facets of the topic, whereas the joint approach would have provided the reader with a more comprehensive and holistic view of the topic. The special issue would have to be virtual, i.e. accepted papers would be published both separately on the different journals they were submitted to, but they would also be collected all together in the final volume of the special issue.

As title for the joint virtual special issue, the publisher proposed "Artificial Intelligence in Breast Cancer Care", and the journals invited to contribute to the virtual special issue were Artificial Intelligence in Medicine (AIM) on the computer-science and methodological side, and The Breast on the clinical and medical side. The editors in chief of the two journals invited the four guest editors to serve in the making of the virtual joint special issue.

The four guest editors had several meetings, where they decided that manuscript submission would have to be by invitation, only. Guest editors also decided that submitted manuscripts should undergo the usual reviewing process every manuscript undergoes when submitted to the respective journals. The four guest editors worked jointly, with the help of the publisher and of the two editors in chief, and they started with the identification of current and major research trends in the field, according to which invitations would have been sent out. Some of the identified trends cover issues shared by both journals, while some other identified trends are mainly covered by only one journal.

Accordingly, the current and major research trends considered include subtopics such as imaging and image analysis; machine learning; decision support systems; big data analytics; natural language processing (NLP). Moreover additional trends have been identified as emerging: personalized medicine; business intelligence; ethical, legal, and social issues.

Once the major research trends were identified, guest editors performed a deep analysis of the corresponding state of the art in order to identify the authors to invite for the joint special issue. This analysis led to 31 invitations on the AIM side and 15 invitations on The Breast side. Sixteen manuscripts were submitted on the AIM side and 13 on The Breast side, leading finally to 9 papers published in the AIM journal and 13 published in The Breast journal.

\section{Declaration of competing interest}

When submitted manuscripts included one of the guest editors as co-author, submissions were managed by the other guest editor (not author of the submission), and outside the official submissionmanagement system of the journal in order to guarantee the blind and peer-review process. Additional details regarding the process followed in compiling the virtual joint special issue is included as online-only supplementary material (link xxxxx).

\section{Acknowledgments}

The construction of the current virtual special issue required much effort from many people. The four guest editors are grateful to: Alison Waldron, Amar Bhogals, Ashley Kieran Clift, David Spencer, Justyna Kasprzycka, Kavitha Narayanasamy, the publishers who launched in 2018 the idea of a joint special issue and constantly helped in the everyday care such an idea required; Fatima Cardoso and Carlo Combi, the editors in chief of the two journals (The Breast and Artificial Intelligence in Medicine) who eagerly accepted the idea; the authors, who enthusiastically accepted the invitations, and carefully revised their manuscripts in guidance of the suggestions and recommendations from reviewers and guest editors; and the anonymous reviewers, whose valuable help set up the pillars on which this project was built.

\section{References}

[1] Coiera E. The price of artificial intelligence. Yearbk Med Inform 2019;28: 14-25. https://doi.org/10.1055/s-0039-1677892.

[2] Ferreira MF, Savoy JN, Markey MK. Teaching cross-cultural design thinking for $\begin{array}{lll}\text { healthcare. } & \text { Breast 2020;50:1-10. https://doi.org/10.1016/ }\end{array}$ j.breast.2019.12.015.

[3] Yap MH, Goyal M, Osman F, Martí R, Denton E, Juette A, Zwiggelaar R. Breast ultrasound region of interest detection and lesion localisation. Artif Intell Med 2020;107(101880). https://doi.org/10.1016/j.artmed.2020.101880.

[4] Carvalho ED, Filho AO, Silva RR, Araújo FH, Diniz JO, Silva AC, ..., Gattass M. Breast cancer diagnosis from histopathological images using textural features and CBIR. Artif Intell Med 2020;105(101845). https://doi.org/10.1016/ j.artmed.2020.101845.

[5] Lee CI, Houssami N, Elmore JG, Buist DS. Pathways to breast cancer screening artificial intelligence algorithm validation. Breast 2020;52:146-9. https:// doi.org/10.1016/j.breast.2019.09.005.

[6] Gullo RL, Eskreis-Winkler S, Morris EA, Pinker K. Machine learning with multiparametric magnetic resonance imaging of the breast for early prediction of response to neoadjuvant chemotherapy. Breast 2020;49:115-22. https:// doi.org/10.1016/j.breast.2019.11.009.

[7] Sechopoulos I, Mann RM. Stand-alone artificial intelligence - the future of breast cancer screening? Breast 2020;49:254-60. https://doi.org/10.1016/ j.breast.2019.12.014.

[8] Tagliafico AS, Piana M, Schenone D, Lai R, Massone AM, Houssami N. Overview of radiomics in breast cancer diagnosis and prognostication. Breast 2020;49: 74-80. https://doi.org/10.1016/j.breast.2019.10.018.

[9] Ibrahim A, Gamble P, Jaroensri R, Abdelsamea MM, Mermel CH, Chen P-HC, Rakha EA. Artificial intelligence in digital breast pathology: techniques and applications. Breast 2020;49:267-73. https://doi.org/10.1016/ j.breast.2019.12.007.

[10] Bessa S, Gouveia PF, Carvalho PH, Rodrigues C, Silva NL, Cardoso F, Cardoso MJ. 3D digital breast cancer models with multimodal fusion algorithms. Breast 2020;49:281-90. https://doi.org/10.1016/j.breast.2019.12.016.

[11] Mavioso C, Araújo RJ, Oliveira HP, Anacleto JC, Vasconcelos MA, Pinto D, ..., Cardoso MJ. Automatic detection of perforators for microsurgical reconstruction. Breast 2020;50:19-24. https://doi.org/10.1016/j.breast.2020.01.001.

[12] Poortmans PM, Takanen S, Marta GN, Meattini I, Kaidar-Person O. Winter is over: the use of Artificial Intelligence to individualise radiation therapy for breast cancer. Breast 2020;49:194-200. https://doi.org/10.1016/ j.breast.2019.11.011.

[13] Cardoso JS, Silva W, Cardoso MJ. Evolution, current challenges, and future possibilities in the objective assessment of aesthetic outcome of breast cancer locoregional treatment. Breast 2020;49:123-30. https://doi.org/10.1016/ j.breast.2019.11.006.

[14] Bouaud J, Pelayo S, Lamy J-B, Prebet C, Ngo C, Teixeira L, ..., Seroussi B. Implementation of an ontological reasoning to support the guideline-based management of primary breast cancer patients in the DESIREE project. Artif Intell Med 2020;101922. https://doi.org/10.1016/j.artmed.2020.101922.

[15] Gu D, Su K, Zhao H. A case-based ensemble learning system for explainable breast cancer recurrence prediction. Artif Intell Med 2020;107(101858). https://doi.org/10.1016/j.artmed.2020.101858.

[16] Macıas-Garcia L, Martınez-Ballesteros M, Luna-Romera JM, Garcia-Heredia JM, Garcia-Gutiérrez J, Riquelme-Santos JC. Autoencoded DNA methylation data to predict breast cancer recurrence: machine learning models and geneweight significance. Artif Intell Med 2020;110(101976). https://doi.org/ 10.1016/j.artmed.2020.101976.

[17] Pozzoli S, Soliman A, Bahri L, Branca RM, Girdzijauskas S, Brambilla M. Domain expertise-agnostic feature selection for the analysis of breast cancer data. Artif Intell Med 2020;101928. https://doi.org/10.1016/j.artmed.2020.101928.

[18] Wang H, Li Y, Khan SA, Luo Y. Prediction of breast cancer distant recurrence using natural language processing and knowledge-guided convolutional neural network. Artif Intell Med 2020;110(101977). https://doi.org/10.1016/ j.artmed.2020.101977.

[19] Butow P, Hoque E. Using artificial intelligence to analyse and teach communication in healthcare. Breast 2020;50:49-55. https://doi.org/10.1016/ j.breast.2020.01.008. 
[20] Moser EC, Narayan G. Improving breast cancer care coordination and symptom management by using AI driven predictive toolkits. Breast 2020;50: 25-9. https://doi.org/10.1016/j.breast.2019.12.006.

[21] Kakileti ST, Madhu HJ, Manjunath G, Wee L, Dekker A, Sampangi S. Personalized risk prediction for breast cancer pre-screening using artificial intelligence and thermal radiomics. Artif Intell Med 2020;105(101854). https://doi.org/ 10.1016/j.artmed.2020.101854.

[22] Chiudinelli L, Dagliati A, Tibollo V, Albasini S, Geifman N, Peek N, ..., Sacchi L. Mining post-surgical care processes in breast cancer patients. Artif Intell Med 2020;105(101855). https://doi.org/10.1016/j.artmed.2020.101855.

[23] Carter SM, Rogers W, Win KT, Frazer H, Richards B, Houssami N. The ethical, legal and social implications of using artificial intelligence systems in breast cancer care. Breast 2020;49:25-32. https://doi.org/10.1016/ j.breast.2019.10.001.

Maria Joao Cardoso* Breast Unit, Champalimaud Clinical Center, Champalimaud Foundation, Lisbon, Portugal

Faculdade de Medicina da Universidade Nova de Lisboa, Nova Medical School, Lisbon, Portugal
Nehmat Houssami Sydney School of Public Health, Faculty of Medicine and Health, Fisher Road, The University of Sydney, New South Wales, Australia

Giuseppe Pozzi

DEIB, Politecnico di Milano, P.za L. da Vinci 32, I-20133, Milano, Italy

Brigitte Séroussi

Sorbonne Université, Université Sorbonne Paris Nord, INSERM, LIMICS UMR_S 1142, F-75006, Paris, France

Assistance Publique - Hôpitaux de Paris, Département de Santé Publique, F-75020, Paris, France

* Corresponding author. Breast Unit, Champalimaud Clinical Center, Champalimaud Foundation, Lisbon, Portugal.

Available online 9 December 2020 